\title{
Macrossismo Virtual: Um Apoio ao Estudo da Sismicidade Brasileira
}

\author{
Larisse Ferreira da Silva ${ }^{1}$, Monique dos Santos Costa ${ }^{1}$, George Sand França1, Juliana Guarrido Damaceno ${ }^{2}$ \\ 1 Observatório Sismológico/UnB \\ 2 Departamento de Geofísica/UFRN
}

Copyright 2016, SBGf - Sociedade Brasileira de Geofísica

Este texto foi preparado para a apresentação no VII Simpósio Brasileiro de Geofísica Ouro Preto, 25 a 27 de outubro de 2016. Seu conteúdo foi revisado pelo Comitê Técnico do VII SimBGt, mas não necessariamente representa a opinião da SBGf ou de seus associados. É proibida a reprodução total ou parcial deste material para propósitos comerciais sem prévia autorização da SBGf.

\section{Abstract}

Recently, the investigation of the records of past earthquakes in Brazil allows us to establish macrosseismic information derived from the study of the historical seismicity, such as earthquakes catalogues and intensity data point's collections. All the technological advances associated with the growth of both information and communication, allows the increased accessibility to the population, enabling the highest record of reports of earthquakes and the effects in a particular locality. This paper presents a contribution to the construction of the Brazilian Macrosseismic Intensity Map, using information shared through digital means.

\section{Introdução}

O rápido desenvolvimento da tecnologia da informação e da comunicação desde meados dos anos 90 e a vontade de investir em inciativas de disseminação de informação, nos permitem termos à nossa disposição uma riqueza de informações sobre a sismicidade de curto a longo prazo através da internet. Essas informações são interpretadas por meio do estudo dos macrossismos que são, por definição, caracterizados como a descrição e medição dos efeitos sísmicos sentidos pela população, por meio de uma escala de intensidade.

O Brasil localiza-se em uma região continental intraplaca estável, apresentando atividade sísmica inferior em relação às bordas de placas (Assumpção et al., 2014). Nesse tipo de região é comum a ocorrência de sismos intraplaca de baixa a média magnitude, causados pelo acúmulo de tensão gerada pelo movimento da placa tectônica. O Brasil pode ser considerado, relativamente, um país assísmico, entretanto com o advento de registros instrumentais, desde o final da década de 70 , foi possível estudar a atividade sísmica brasileira. Uma das regiões brasileiras mais ativas é o nordeste, nos estados do Ceará, Rio Grande do Norte e Pernambuco (Berrocal et al., 1984; França, 2006).

Em 2014, houve uma expansão da Rede Sismográfica Brasileira (RSBS), principalmente em relação às regiões Centro-Oeste e Norte do país, antes caracterizadas pela baixa densidade de estações sismográficas ocasionadas pela sua grande área de extensão, dificuldade de acesso e logística e falta de equipamentos necessários (Rocha et al., 2015). Nessas condições havia muita dificuldade em registrar eventos de baixa magnitude, tornando o processo de relatos macrossísmicos, ainda, em uma importante ferramenta para o registro da sismicidade histórica brasileira.

O objetivo desse estudo é registrar e classificar os dados macrossísmicos, investigando seus efeitos no território brasileiro, desde os centros urbanos até as localidades rurais. Com essas informações, podemos contribuir para a construção do Mapa de Intensidade Macrossísmica do Brasil e realizar uma discussão em torno das dificuldades relacionadas à obtenção e classificação dos dados.

\section{Metodologia}

Os dados foram coletados a partir da divulgação de notícias em jornais online, blogs, além de relatos da população em redes sociais como o Twitter e o Facebook, durante o período de janeiro de 2006 a março de 2016, totalizando 362 eventos analisados, com média de 3 notificações por mês (Figura 1). Os eventos foram notificados por meio da atribuição de palavras chaves, relacionadas à atividade sísmica, a partir de ferramentas como o Google Alert. Posteriormente, esses dados foram selecionados e postos em uma planilha, onde foram classificados como Eventos Naturais, Andinos, Artificiais ou Outros. Além disso, foram informados a data, a hora (UTC), as coordenadas do epicentro e a magnitude do evento, bem como quais veículos de informação foram responsáveis pela notificação e os informes básicos sobre os relatos da população e os danos materiais nas regiões de influência. A partir desses relatos foi possível estimar a intensidade máxima de cada evento, com base na Escala Modificada de Mercalli (MM). No decorrer da análise, foi notável a presença recorrente de sismos com epicentro na região Andina, sentidos pela população brasileira.

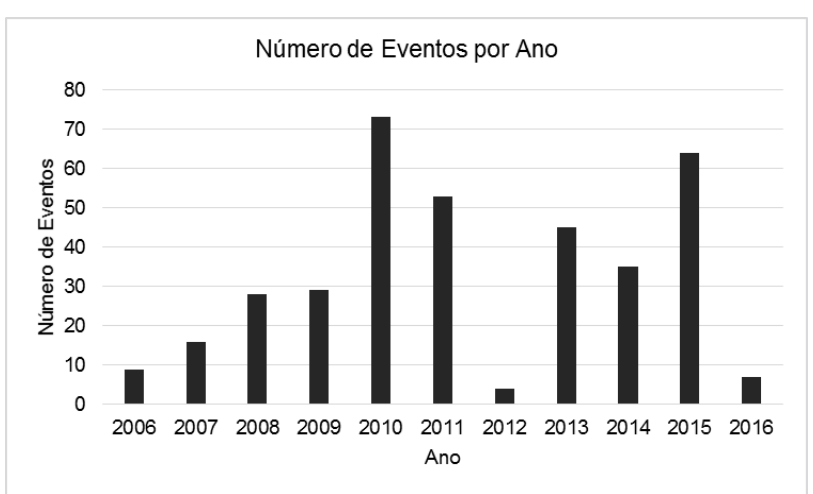

Figura 1 - Histograma mostrando a relação do número de eventos analisados por ano, no período de 2006 a 2016. 


\section{Resultados}

A partir das informações coletadas foi possível gerar um mapa de macrossismo com intensidade dos eventos (Figura 3a). A nível de comparação e complementação, foi utilizado um mapa de magnitude $\left(m_{R}\right)$, fornecido pelo Observatório Sismológico (SIS) da Universidade de Brasília (UnB) (Figura 3b). A partir da visualização dos mapas, nota-se a concentração majoritária de eventos notificados nas regiões nordeste e sudeste brasileiros.

A Figura 2a revela a maior incidência de eventos com intensidade III, segundo a Escala Modificada de Mercalli. Já a Figura $2 \mathrm{~b}$ relaciona a quantidade de eventos por magnitude $\left(m_{R}\right)$, lembrando que baixas magnitudes são difíceis de serem determinadas com precisão.
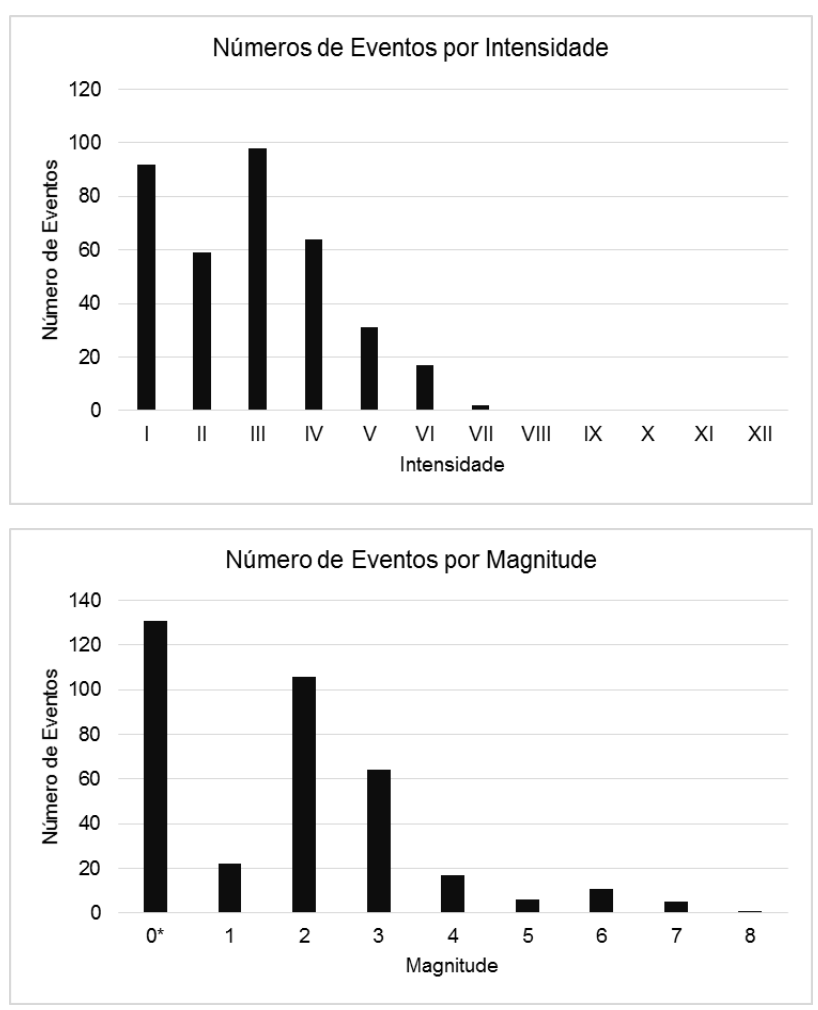

Figura 2 - (a) Histograma do número de sismos analisados por intensidade e (b) por magnitude. *Os valores entre zero e um não foram determinados.

Nesse período houveram 262 eventos classificados como naturais. A média das magnitudes dos eventos registrados foi de $2.5 \mathrm{~m}_{\mathrm{R}}$, considerada baixa. Já a média da intensidade sísmica foi de III, revelando que os efeitos nas construções civis, como rachaduras e objetos balançando, são os principais efeitos relatados pela população. A maioria dos sismos ocorreram na cidade de Sobral (CE), seguidamente por Pedra Preta (RN) e Caruaru (PE), confirmando a maior atividade sísmica na região nordeste brasileira.
Os eventos classificados como Andinos, também são naturais, porém são provenientes de regiões com distância epicentral acima de $1500 \mathrm{~km}$. Nesse caso, foram registrados 22 eventos na região dos Andes, onde a atividade sísmica é marcada pela subducção entre as placas Nazcar e Sul-americana (Bilek, 2010). A média das magnitudes foi de $5.5 \mathrm{mb}$, considerada alta para os padrões brasileiros, enquanto a média das intensidades foi III, sendo considerada baixa. Isso acontece porque, na maioria das vezes, os efeitos sentidos no Brasil, ocorrem em lugares altos como, por exemplo, em prédios devido à amplificação das ondas sísmicas, como no caso de São Paulo, em setembro de 2015. Em contraponto, as maiores intensidades sentidas ocorreram nas regiões brasileiras ao extremo oeste, localizadas principalmente na região norte, como aconteceu nos estados do Acre, Rondônia e Amazonas em novembro de 2015.

Eventos artificiais, exemplificados por explosões de mineradoras, construção de estradas e estruturas de prédios, totalizaram 34 casos no catálogo. A média das magnitudes, $2.0 \mathrm{~m}_{\mathrm{R}}$, e das intensidades, III, dos sismos, podem ser confundidos facilmente com os eventos classificados como naturais. Em relação aos eventos classificados como Outros, foram registrados 44 casos relatados pela população onde, entretanto, não houveram confirmação de sismos naturais ou de explosões locais.

\section{Discussão e Conclusões}

Ao longo da análise dos dados foi observada a dificuldade em determinar a intensidade dos eventos. Isso nos remete a uma problemática, pois, por mais que a Escala Modificada de Mercalli seja a mais utilizada mundialmente para a determinação da intensidade sísmica, originalmente a mesma foi criada com base em países europeus. Isso quer dizer que, não é a mesma coisa comparar, por exemplo, a estrutura das construções civis europeias às brasileiras. Antes disso, devemos analisar o modo como cada indivíduo reage perante a uma situação de abalo sísmico. Como no Brasil a ocorrência de tremores é consideravelmente baixa em relação aos países localizados em zonas de alta atividade sísmica, a população não está adaptada ao fenômeno em si e seus efeitos no espaço. Esse tipo de reação é notável quando telessismos andinos são sentidos, no alto de prédios, principalmente na região sudeste brasileira. Assim, surge uma possível proposta para a criação de uma nova escala de intensidade, menos subjetiva e visando o território brasileiro como algo a se levar em conta. Além disso, deparamo-nos com a necessidade da criação de um formulário padronizado, a fim de tornar 0 processo de classificação da intensidade sísmica, mais confiável e menos subjetivo.

Ao final da análise, foi possível romper o paradigma de que não há tremores no Brasil. $\mathrm{Na}$ verdade, observa-se uma quantidade significativa a ponto de ser necessário o desenvolvimento de estudos e, consequentemente, medidas preventivas por parte de órgãos públicos, deliberados pela Defesa Civil. 

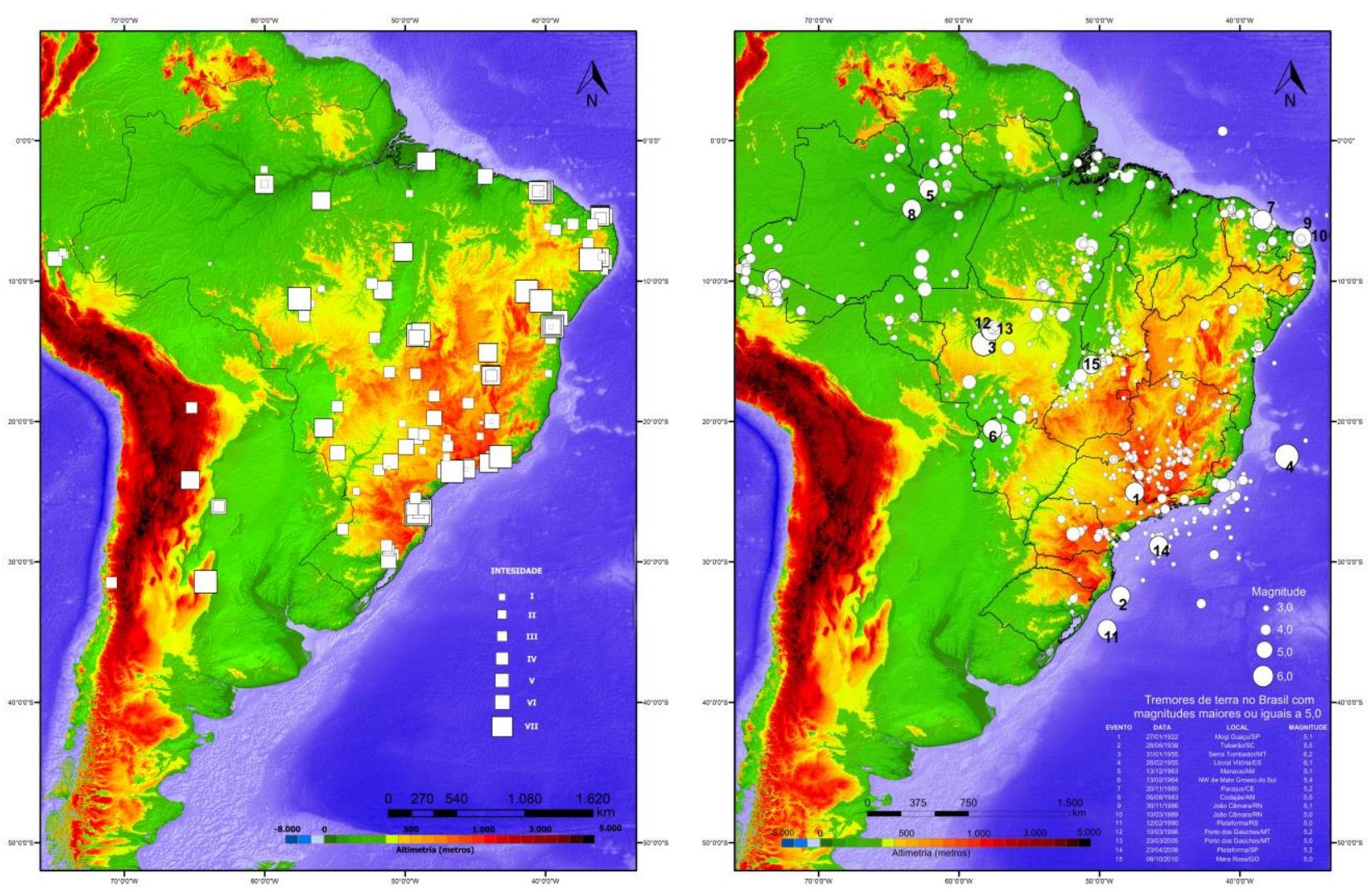

Figura 3 - (a) Mapa de macrossismo com a intensidade sísmica no período de 2006-2016 e (b) mapa de magnitude fornecido pelo SIS-UnB.

\section{Agradecimentos}

Ao Observatório Sismológico da Universidade de Brasília, pelo suporte oferecido ao desenvolvimento da pesquisa, e ao lago Guilherme dos Santos Lemos, pelo apoio técnico.

\section{Referências}

Assumpção, M. S., Ferreira, J., Barros, L. V., Bezerra, H., França, G. S., Barbosa, J. R., Menezes, E., Ribotta, L. C., Prichiner, M., Nascimento, A., Dourado, J. C., 2014. Intraplate seismicity in Brazil. In: TALWANI, Preadeep. Intraplate earthquakes. São Paulo: Cambridge University Press. Cap.3, p. 50-71.

Berrocal, J., Assumpção, M. S., Antezana, R., Dias Neto, C. M., Ortega, R., Franca, H., Veloso, J. A. V. 1984. Sismicidade do Brasil. Instituto Astronómico e Geofísico, São Paulo, Brasil, 320p.

França, G. S. 2006. Brazil Seismicity. Bulletin of the International Institute of Seismology and Earthquake Engineering, Japão, v. 40, p. 23-36.
Rocha, M. P., Assumpção, M., Carvalho, J., Barbosa, J., França, G., Bianchi, M., Barros, L., Azevedo, P. A., Collaco, B., 2015. Intensificação do monitoramento sismológico nas Regiões Centro-Oeste e Norte do país pela implantação da Rede Sismográfica Brasileira (RSBR). In: XV Simpósio Nacional de Estudos Tectônicos, 2015, Vitória - ES. Simpósio Nacional de Estudos Tectônicos, 2015.

Bilek, S.L., 2010. Seismicity along the South American subduction zone: Large earthquakes, tsunamis, and subduction zone complexity (invited review paper), Tectonophysics, 495, 2-14. 